\title{
Multiple independent origins for a subtelomeric locus associated with growth rate in Fusarium circinatum
}

\author{
Stephanie Van Wyk, Brenda D. Wingfield, Lieschen De Vos, Quentin C. Santana, Nicolaas A. Van der Merwe, and Emma T. \\ Steenkamp
}

Department of Biochemistry, Genetics and Microbiology, Forestry and Agricultural Biotechnology Institute (FABI), University of Pretoria, Private Bag X20, Pretoria, 0028, South Africa; corresponding author email: emma.steenkamp@fabi.up.ac.za

\begin{abstract}
Fusarium is a diverse assemblage that includes a large number of species of considerable medical and agricultural importance. Not surprisingly, whole genome sequences for many Fusarium species have been published or are in the process of being determined, the availability of which is invaluable for deciphering the genetic basis of key phenotypic traits. Here we investigated the distribution, genic composition, and evolutionary history of a locus potentially determining growth rate in the pitch canker pathogen $F$. circinatum. We found that the genomic region underlying this locus is highly conserved amongst $F$. circinatum and its close relatives, except for the presence of a 12000 base pair insertion in all of the examined isolates of $F$. circinatum. This insertion encodes for five genes and our phylogenetic analyses revealed that each was most likely acquired through horizontal gene transfer from polyphyletic origins. Our data further showed that this region is located in a region low in $\mathrm{G}+\mathrm{C}$ content and enriched for repetitive sequences and transposable elements, which is situated near the telomere of Chromosome 3 of $F$. circinatum. As have been shown for other fungi, these findings thus suggest that the emergence of the unique 12000 bp region in F. circinatum is linked to the dynamic evolutionary processes associated with subtelomeres that, in turn, have been implicated in the ecological adaptation of fungal pathogens.
\end{abstract}

Key words:

Fusarium temperatum

horizontal gene transfer

Pitch canker fungus

transposable elements

Article info: Submitted: 12 January 2018; Accepted: 19 February 2018; Published: 27 February 2018.

\section{INTRODUCTION}

Fusarium species are remarkably diverse (Leslie \& Summerell 2006, O'Donnell et al. 2013). Despite the extensive genomic synteny characterizing this genus (Waalwijk et al. 2004, Ma et al. 2010, Lysøe et al. 2014), individual species are not only phenotypically complex but also display a wide range of species-specific traits (Wiemann et al. 2013, Herron et al. 2015, Sperschneider et al. 2015). Comparative studies are increasingly showing that this diversity also extends to their genomic architectures and genetic content (Waalwijk et al. 2004, De Vos et al. 2011, Chiara et al. 2015, Hansen et al. 2015). For example, the closely related species $F$. circinatum and $F$. temperatum are characterized by substantial levels of both macro- and micro-synteny (De Vos et al. 2014), but they are, respectively, pathogens of pine (Hepting \& Roth 1946, Leslie et al. 2006) and maize (Scauflaire et al. 2011). They also differ dramatically in other phenotypic traits (Desjardins et al. 2000, De Vos et al. 2007, 2011), including growth rate for which a major Quantitative Trait Locus (QTL) has previously been identified (De Vos et al. 2011).

Certain parts of Fusarium genomes appear to be more variable than others (Cuomo et al. 2007, Coleman et al. 2009, Ma et al. 2010, Chiara et al. 2015, Sperschneider et al. 2015). In addition to the telomeres and centromeres (Chiara et al. 2015, Sperschneider et al. 2015), areas of high sequence variability also occur in other chromosomal regions and may even extend across entire chromosomes such as the supernumerary or dispensable chromosomes (Ma et al. 2010, Van der Nest et al. 2014). Generally, these variable regions in diverse fungi are rich in repeats and transposable elements (TEs), have $G+C$ contents that differ markedly from the rest of the genome (Goodwin et al. 2011), and often encode nonessential genes (Fedorova et al. 2008, Coleman et al. 2009, Sperschneider et al. 2015). Overall, such regions of variability are thought to accelerate genome evolution and plasticity and to promote adaptation (Fedorova et al. 2008, Coleman et al. 2009, Chiara et al. 2015).

The genomes of filamentous fungi are dynamic and capable of tolerating extensive gene gains and losses (Braun et al. 2000, Coleman et al. 2009, Spanu et al. 2010, Raffaele \& Kamoun 2012). Gene gains may occur via internal genomic mutations (i.e. intra-genomic mutations) due to duplication, displacement and translocation events (Gac \& Giraud 2008, Proctor et al. 2009, De Vos et al. 2014), or via gene introductions from external sources through horizontal gene transfer (HGT) (Ma et al. 2010, Chuma et al. 2011, Hansen et al. 2015). HGT refers to the exchange of

\section{2018 International Mycological Association}

You are free to share - to copy, distribute and transmit the work, under the following conditions:

Attribution: $\quad$ You must attribute the work in the manner specified by the author or licensor (but not in any way that suggests that they endorse you or your use of the work).

Non-commercial: $\quad$ You may not use this work for commercial purposes.

No derivative works: You may not alter, transform, or build upon this work.

For any reuse or distribution, you must make clear to others the license terms of this work, which can be found at http://creativecommons.org/licenses/by-nc-nd/3.0/legalcode. Any of the above conditions can be waived if you get permission from the copyright holder. Nothing in this license impairs or restricts the author's moral rights. 
genetic material between different strains or species, which would include those due to hybridization (Brown \& Doolittle 1999). Nevertheless, such gains and differential losses have apparently given rise to species-specific regions in various fungi (Daboussi \& Capy 2003, Coleman et al. 2009, Proctor et al. 2009, Ma et al. 2010, Spanu et al. 2010), e.g. lineages of Magnaporthe, Aspergillus, Fusarium and Coccidioides (Galagan et al. 2005, Behnsen et al. 2008, Skamnioti et al. 2008, Coleman et al. 2009, Proctor et al. 2009, Moran et al. 2011, Hansen et al. 2015). Recently it was also demonstrated that such gains and losses have been particularly important in driving the formation of species-specific regions within the telomeric regions of certain Fusarium species (Chiara et al. 2015).

The acquisition of genes via HGT is regarded as an important and ongoing source of functional novelty in fungi (Ma et al. 2010, Wisecaver et al. 2014, Jaramillo et al. 2015). Compared to other eukaryotes, and some prokaryotes (Nelson et al. 1999, Crisp et al. 2015), this form of gene gains is relatively high in fungi (Gardiner et al. 2013, Glenn et al. 2016). This is also true for Fusarium species, where HGTs are thought to have shaped their evolution and contributed to the emergence of species-specific traits (Ma et al. 2010, Alves et al. 2014, Sieber et al. 2014, Stewart et al. 2014, Wisecaver et al. 2014, Glenn et al. 2016). For example, F. graminearum, $F$. verticillioides and $F$. oxysporum f. sp. lycopersici have species-specific gene clusters that were likely acquired across species boundaries (Sieber et al. 2014, Glenn et al. 2016). In F. verticillioides it was also recently shown that certain gene clusters were acquired from multiple external sources as opposed to having been acquired through gene duplication and differential gene loss (Stewart et al. 2014, Glenn et al. 2016).

In this study, we examined the chromosomal location and evolutionary origins of the major QTL associated with growth rate variation in $F$. circinatum, that was previously identified in a genetic linkage map of an interspecific cross between F. circinatum and F. temperatum (De Vos et al. 2007, 2011). For this purpose, our study had four specific objectives. Firstly, we located the genetic marker linked to growth rate variation (i.e. marker AT/AC-625bh) in the genome of $F$. circinatum (Wingfield et al. 2012) and identified the genes encoded in the region underlying it by making use of various in silico approaches. Secondly, the identified region and the chromosomal areas surrounding it were examined in terms of the likely functions they encode, their $\mathrm{G}+\mathrm{C}$ content, and the presence and distribution of repeats and TEs. Thirdly, the presence and distribution of the region identified was assessed in a broad collection of Fusarium species and in other isolates of $F$. circinatum by making use of PCR-based analyses and genome-based searches. For the latter, the two F. circinatum genomes already in the public domain (Wingfield et al. 2012, Van der Nest et al. 2014) were supplemented by sequencing the genome for a third isolate obtained from diseased pine seedling roots in South Africa (Steenkamp et al. 2014). Finally, the putative origin of the identified region was evaluated using various sequence alignments and phylogenetic analyses. These fine-scale synteny comparisons and phylogenetic information revealed genetic features that likely facilitated the emergence of a phenotype- associated QTL and further broaden our understanding of genetic differentiation amongst related fungal lineages.

\section{MATERIALS AND METHODS}

\section{Genome sequencing and assembly}

Fusarium circinatum isolate KS17 (CMW 674; Culture collection of the Forestry and Agricultural Biotechnology Institute, FABI, University of Pretoria, South Africa) was obtained from the infected root tissue of a Pinus radiata seedling collected in a nursery in the Western Cape, South Africa in 2005 (Steenkamp et al. 2014). The isolate was grown in half strength potato dextrose broth $(20 \% \mathrm{w} / \mathrm{v})$ and incubated at $25^{\circ} \mathrm{C}$ in the dark on an orbital shaker at $120 \mathrm{rpm}$ for $7 \mathrm{~d}$, after which DNA was extracted (Möller et al. 1992). The DNA was used to prepare two mate-pair libraries (1000 base pair [bp] insert size) and a single-read library, which were then sequenced by SEQOMICS (Csongrád, Hungary) using the SOLiDTM V4 technology (Applied Biosystems, California, USA) producing reads containing ca. 50 bp. Sequence reads were quality filtered using CLC Genomics Workbench v.8.0 (CLCbio, Aarhus, Denmark), assembled into scaffolds using ABySS v.1.5.2 (Simpson et al. 2009), after which gapped regions within scaffolds were closed with GapFiller v1.11 (Boetzer \& Pirovano 2012). Completeness of the genome assembly was evaluated with BUSCO v.2.0.1 using the Sordariomycetes gene set (Simão et al. 2015). WebAUGUSTUS (Hoff \& Stanke 2013) to predict putative open reading frames (ORFs) based on the gene models for $F$. graminearum and mRNA data from $F$. circinatum (Wingfield et al. 2012).

\section{Genomic localization of marker AT/AC-625bh, a major growth rate determining QTL in $F$. circinatum}

The location of marker AT/AC-625bh (De Vos et al. 2011) within the genome sequence of isolate FSP34 of $F$. circinatum (Wingfield et al. 2012) was determined as described previously (De Vos et al. 2014). This was done with in silico Amplified Fragment Length Polymorphism (AFLP) analysis using AFLPinSilico v2 (Rombauts et al. 2003), which involved the use of simulated restriction enzyme digestion profiles for the entire genome of $F$. circinatum. The analysis used the restriction sites for EcoRI (GAATT $\downarrow C$ ) and Msel (TTA $\downarrow A$ ) with an adapter length of zero, as well as $A C$ and $A T$ selective nucleotides (De Vos et al. 2007). In order to account for initial variability in estimated restriction fragment sizes, all restriction fragments in the size range 595-635 bp were considered in the analysis. By making use of nucleotide BLAST (Basic Local Alignment Search Tool; Altschul et al. 1997) searches and alignments in CLC Main Workbench software (CLC Bio-Qiagen, Aarhus, Denmark, version 7.0.3), sequences of the in silico restriction fragments were then compared to those in the most recent version of the published assembly of F. circinatum (Wingfield et al. 2012). The latter was represented in the genome database of the National Centre for Biotechnology Information (NCBI; http://www. ncbi.nlm.nih.gov/) by a draft pseudo-chromosome assembly (BioProject PRJNA41113) with accession AYJV00000000.2. 


\section{Sequence characterization of the genomic region containing marker AT/AC-625bh}

The stretch of sequence containing marker AT/AC-625bh, as well as regions up- and downstream of it were characterized in terms of $\mathrm{G}+\mathrm{C}$ content and the occurrence and distribution of repetitive elements. The $G+C$ content was determined using CLC Genomics Workbench and a sliding window of $1000 \mathrm{bp}$ and step size of $500 \mathrm{bp}$. For identifying repeat elements, Repeat Masker (Tarailo-Graovac \& Chen 2009) and Tandem Repeat Finder (Benson 1999) were used. Putative transposable elements (TE) were identified by using the CENSOR-EMBL fungal TEs database (Kohany et al. 2006, Li et al. 2015). Repeat and TE density were determined using a sliding window of $1000 \mathrm{bp}$ with $500 \mathrm{bp}$ increments. In order to determine the abundance of the telomere-associated repeat sequence "TTAGGG/CCCTAA" (Garcia-Pedrajas \& Roncero 1996, Fulnečková et al. 2013), a motif search was conducted in CLC Genomics Workbench using a sliding window of 1000 bp with 500 bp increments. All repeats showing $80 \%$ similarity to the telomere-associated sequence were considered in this analysis.

The functions of genes encoded on the stretch of genome sequence containing marker AT/AC-625bh were also inferred. This was done using InterProScan (Zdobnov \& Apweiler 2001) to determine Gene Ontologies (GO), protein family membership (PFAM) and protein functional domains. Putative secondary metabolism gene clusters were identified using Antibiotics and Secondary Metabolites Analysis Shell (antiSMASH) (Blin et al. 2013). Gene density was estimated using a window size was $10000 \mathrm{bp}$ and the step size 5000 bp.

\section{Synteny analysis of the genomic region containing marker AT/AC-625bh}

Synteny and collinearity across the region containing marker AT/AC-625bh were evaluated using nucleotide alignments of the relevant genomic sections in representative Fusarium isolates and species (Supplementary Table S1). Together with the genome data for $F$. temperatum and F. circinatum isolate FSP34, we also included those for two additional isolates of F. circinatum KS17 (this study) and GL1327 (Van der Nest et al. 2014) as well as additional taxa in the F. fujikuroi species complex (FFSC) (Geiser et al. 2013); i.e. F. verticillioides (Cuomo et al. 2007), F. mangiferae (Niehaus et al. 2016) and F. fujikuroi (Wiemann et al. 2013). For comparison we also included representatives of other well-known Fusarium complexes; i.e. F. graminearum (Cuomo et al. 2007), F. oxysporum (Ma et al. 2010) and F. solani (Coleman et al. 2009).

These genome-based synteny and collinearity analyses were complemented with PCRs and Sanger sequencing. This was done to confirm the assembly of the genomic region containing marker AT/AC-625bh in 22 diverse isolates of F. circinatum (Supplementary Table S1). The approach was also used to confirm breaks in synteny and collinearity in representative isolates of other Fusarium species. All primers (Supplementary Table S2) were designed using Primer3 (Untergasser et al. 2012). Genomic DNA was extracted from each isolate using a previously described protocol (Steenkamp et al. 1999). All amplification reactions were performed using MyTaq ${ }^{\mathrm{TM}}$ DNA polymerase (Bioline Reagents Ltd., MA), according to the supplier's protocol. Purified PCR products were sequenced at the Department of Genetics at the University of Pretoria, using the ABI 3500xl Genetic Analyzer (Applied Biosystems, CA).

\section{Putative origins of the genomic region containing marker AT/AC-625bh}

For each of the genes encoded in the genomic region containing the QTL marker, a dataset of homologous protein sequences was assembled. The sequences included in these datasets were identified using BLAST searches against eight publically available Fusarium genomes (Supplementary Table S3), as well as the genome databases of MycoCosm (Grigoriev et al. 2013) (Joint Genome Institute [JGI], US Department of Energy) and the NCBI. For the latter, query sequences were searched against those in the non-redundant database using the online position-specific iterative (psi) BLAST tool (Altschul et al. 1997). In order to exclude highly divergent protein sequences, we only considered those BLAST sequences with at least $40 \%$ amino acid identity over $70 \%$ of the query sequence length and that had expect-values $[E]<1 \times 10^{-5}$ and bit scores $>200$. Also, predicted proteins classified as "partial proteins" were excluded, and only the fully predicted proteins were considered for further analyses.

Individual sequence datasets were aligned using $\mathrm{CO}$ BALT (Constraint-based Multiple Protein Alignment Tool) (Papadopoulos \& Agarwala 2007) with default settings (https:// www.ncbi.nlm.nih.gov/tools/cobalt/re_cobalt.cgi).These alignments were then trimmed in BioEdit v.7.0.9.0 (Hall 1999) to ensure that all of the sequences spanned the same region. Each dataset was subjected to ProtTest 3.2 (Abascal et al. 2005) to determine the best-fit substitution model. These model parameters were then used to perform Maximum Likelihood phylogenetic analyses with MEGA 6.0 (Tamura et al. 2013). Branch support was evaluated using the same model parameters and 1000 bootstrap pseudo-replicates.

Relative to the overall phylogenetic relationships among the FFSC species and its Fusarium relatives, we also investigated the relationships between the F. circinatumspecific genes encoded in the AT/AC-625bh-containing region to those encoded elsewhere in the examined Fusarium genomes (Supplementary Table S3). Dataset construction and phylogenetic analyses were performed as described above, except that BLASTp was used to identify homologs and only full-length sequences were included. Another round of analyses was also conducted where we constructed overview trees of the top BLAST hits (irrespective of bit scores and query coverage) in the NCBI databases using a neighbor-joining approach in MEGA.

\section{RESULTS}

\section{Fusarium circinatum isolate KS17 genome sequence}

The draft genome assembly of $F$. circinatum isolate KS17 was $46325048 \mathrm{bp}$ in size. It had an average coverage of $166 x$ and $G+C$ content of $44.69 \%$. The assembly consisted of 6033 contigs (>200bp) with an N50-value of 95695 bp. 
BUSCO suggests that the assembly was $76.2 \%$ complete (i.e. complete BUSCOs $=76.2 \%$; complete and single-copy BUSCOs $=75.1 \%$; complete and duplicated BUSCOs = $1.1 \%$; fragmented BUSCOs = $17.0 \%$; missing BUSCOs = $6.8 \%$; number of BUSCOs searched $=3725$ ) (Simão et al. 2015). WebAUGUSTUS predicted that it encodes 16502 putative ORFs. The F. circinatum KS17 genome sequence data were deposited at DDBJ/EMBL/GenBank under the accession number LQBB00000000. The version described here is version LQBB01000000.

\section{Genomic localization of marker AT/AC-625bh}

In silico AFLP analysis and sequence comparisons (De Vos et al. 2011) revealed that marker AT/AC-625bh is $599 \mathrm{bp}$ in size. It was located within the gene FCIRG_04559 of $F$. circinatum (FSP34). Marker AT/AC-625bh was positioned from nucleotides $39762-40361$ on Chromosome 3 (NCBI accession CM004513.1). Note that this corresponds to position 9 351-9 950 on contig 02138 of the previous version of the assembly (Wingfield et al. 2012)

\section{Sequence characterization of the genomic region containing marker AT/AC-625bh}

The first 100000 bp of Chromosome 3 of $F$. circinatum that contained marker AT/AC-625bh was characterized further. Based on InterProScan, this region encoded a diverse range of putative protein products (Supplementary Tables S4 and S5). However, it appeared to be enriched for those involved in transmembrane substrate transportation (FCIRG_04551 and FCIRG_04555), transcriptional regulation (FCIRG_04552, FCIRG_04556 and FCIRG_04559), carbon metabolism (FCIRG_04550 and FCIRG_04553), and catalytic activities (FCIRG_04549, FCIRG_04553, FCIRG_04557, FCIRG_04558). The analysis with antiSMASH also predicted the presence of a biosynthetic gene cluster between 53 928-81 890bp (Supplementary Table S6; FCIRG_03382, FCIRG_03383, FCIRG_03384, FCIRG_03385 and FCIRG_03388) with similarity to the gene cluster involved in butirosin biosynthesis.

Large changes in $\mathrm{G}+\mathrm{C}$ content, gene, TE and repeat density were found across the examined portion of Chromosome 3 (Fig. 1). Based on $G+C$ content, the first 12 $000 \mathrm{bp}$ were markedly different from the remainder of the sequence. After averaging ca. $27 \%$ in the first $12000 \mathrm{bp}$, the $\mathrm{G}+\mathrm{C}$ content increased to an average of ca. $48.5 \%$. In terms of gene density, this first section also encoded fewer genes compared to the rest of Chromosome 3. We observed a similar distribution pattern for the repeats (Supplementary Table S7-S8) and putative TEs (Supplementary Table S9 and Supplementary Fig, S1), which were notably more abundant in the first $14000 \mathrm{bp}$ compared to that of the remainder of the downstream regions. The same was also true for the telomerespecific "TTAGGG" repeat motif (Supplementary Fig. S2). Therefore, based on $\mathrm{G}+\mathrm{C}$, repeat, TE (Supplementary Fig. S1) and gene content, marker AT/AC-625bh is located in the subtelomeric region of Chromosome 3 of $F$. circinatum.

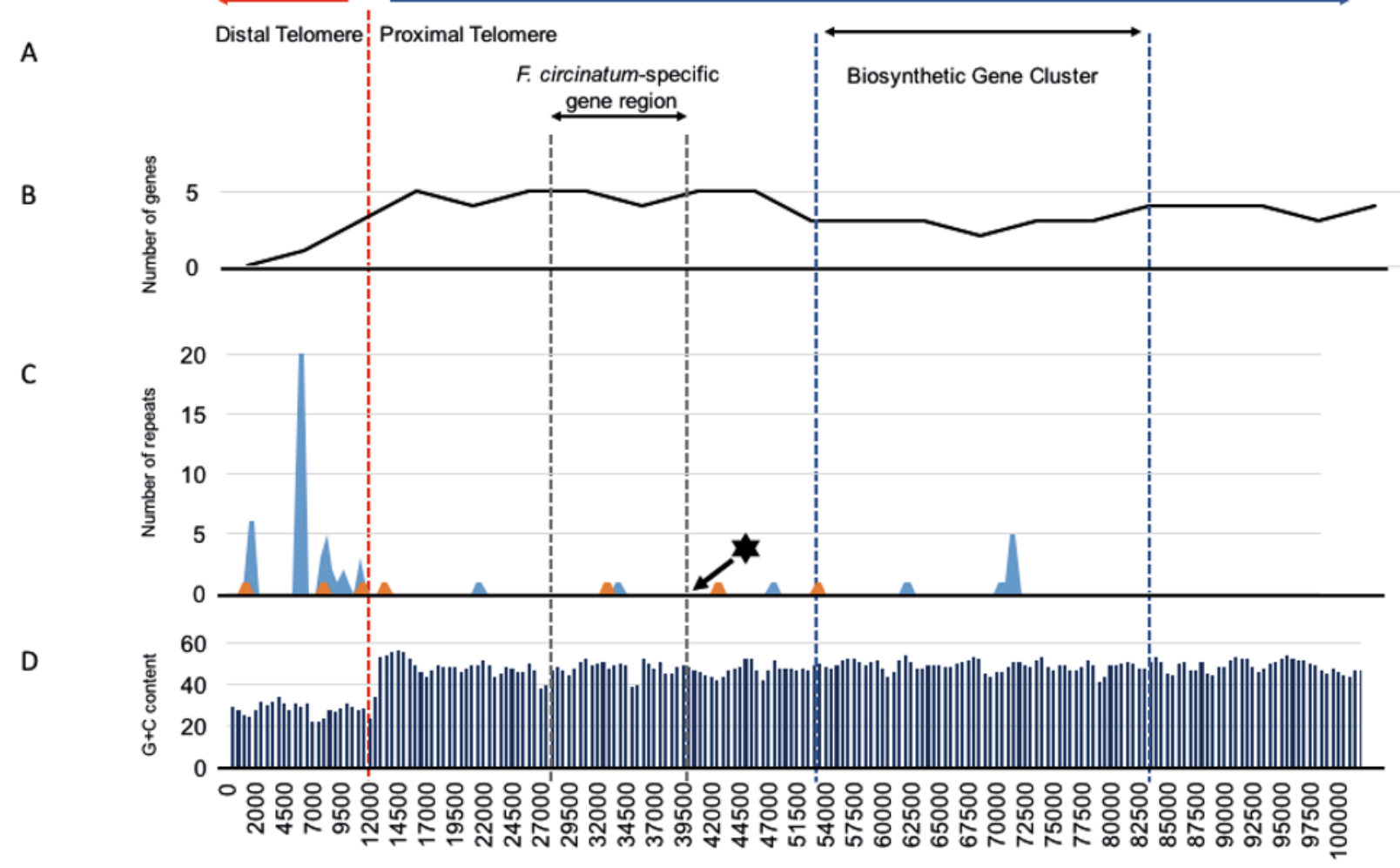

Genomic position

Fig. 1. Genomic features of the first 100000 bp of Chromosome 3 of Fusarium circinatum (FSP34). (A) This region corresponds to the subtelomere of the chromosome. (B) Line graph illustrating the change in gene count determined through a 10000 bp sliding window at 5000 bp increments. (C) Chart showing the count of simple repeat and tandem repeat sequences in blue and the count of transposable element associated repeat sequences in orange; these were determined using a $10 \mathrm{bp}$ sliding window at $500 \mathrm{bp}$ increments, and the black star indicates the position of the QTL marker. (D) The data series represents G+C (\%) content, which was determined with a 1000 bp sliding window at 500 bp increments. 


\section{Synteny analysis of the genomic region containing marker AT/AC-625bh}

We first compared the gene content and orientation of the region containing the AFLP marker in the genome of $F$. circinatum FSP34 to those in the two other F. circinatum genomes (i.e. for isolate KS17 and GL1327). All 15 genes encoded in the region containing marker AT/AC-625bh were present in the same order and orientation in these three genomes. The intergenic PCR and Sanger sequencing analysis of this region, in 21 additional isolates of the fungus, further confirmed the genomic assembly of this region, as well as the order and orientation of genes (results not shown).

Subsequent interspecies comparisons revealed that the ca. 12000 bp region containing marker AT/AC-625bh was absent from the corresponding genomic regions in other Fusarium species (Fig. 2). This 12000 bp sequence encode five genes (FCIRG_04559, FCIRG_04558, FCIRG_04557, FCIRG_04556 and FCIRG_04555). This genome-based observation was confirmed with PCR and Sanger sequencing, where our primers were designed to span the synteny breakpoint (i.e. from the end of gene FCIRG_04560 to the start of gene FCIRG_04554). These analyses confirmed that the $12000 \mathrm{bp}$ region was indeed absent from the genomes of the other FFSC species examined (i.e. F. temperatum, F. mangiferae, F. fujikuroi and F. verticillioides). However, genome-based comparisons of the up- and downstream regions flanking the $12000 \mathrm{bp}$ insert in $F$. circinatum, revealed a high degree of conserved synteny amongst the FFSC species included. This homology also extended to the sequenced representatives of $F$. oxysporum (Supplementary Table S10), but not to the more distantly related F. graminearum and F. solani (Supplementary Table S11).

\section{Putative origins of the genomic region containing marker AT/AC-625bh}

To examine the potential origins of the AT/AC-625bh markercontaining region specific to $F$. circinatum, the five genes encoded on this 12000 bp stretch of DNA were compared to those included in various local and public databases. This allowed for the identification of homologous proteins for all five of the genes encoded in this $F$. circinatum-specific region (Supplementary Tables S12-S15). However, none of the five genes co-occurred (i.e., located together on the same contig or chromosome) in any of the fungal genomes examined. Furthermore, the taxa with which the F. circinatum-specific sequences shared identity differed markedly among the five genes.

Phylogenetic analysis of datasets containing only Fusarium sequences revealed that none of FCIRG_04559, FCIRG_04558, FCIRG_04557, FCIRG_04556 and FCIRG_04555 grouped with other sequences from $F$. circinatum (Supplementary Fig. S3). The same pattern was observed in the overview trees inferred from the top BLAST hits for each gene in the NCBI database (Supplementary Fig. S4). This was also true even if the FSP34 genome contained a second homolog of the gene, as is the case for FCIRG_04559 and FCIRG_04557. In both instances, the gene encoded in the $F$. circinatum-specific region did not group with F. circinatum or other members of the FFSC. None of the five genes in the $F$. circinatum specific region was thus characterized by a phylogeny matching that expected for the FFSC.

Rigorous phylogenetic analyses of the F. circinatumspecific region revealed that the genes in this locus have distinct evolutionary ancestries (Fig. 3). Based on these results, FCIRG_04556 and FCIRG_04559 were most closely related to proteins encoded by $F$. solani. In the phylogenetic trees containing homologs of FCIRG_04555

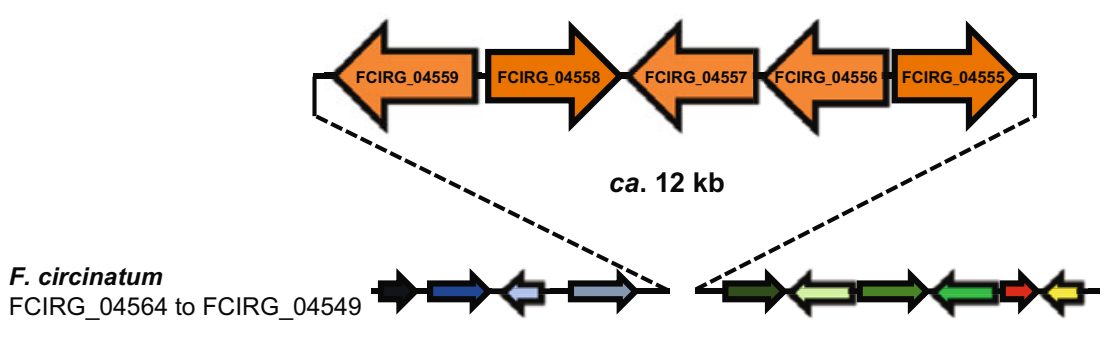

F. temperatum

DC32_4595 to DC32_4602

F. verticillioides

FVEG12543 to FVEG12550

F. fujikuroi

FFUJ02128 to FFUJ02140

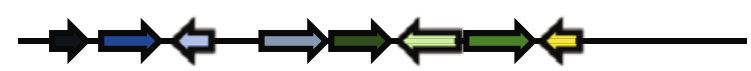

F. mangiferae

FMAN03868 to FMAN03877

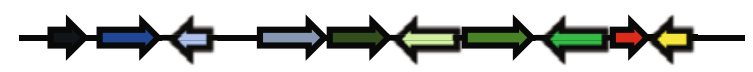

F. oxysporum

FOXG15227 to FOXG15219
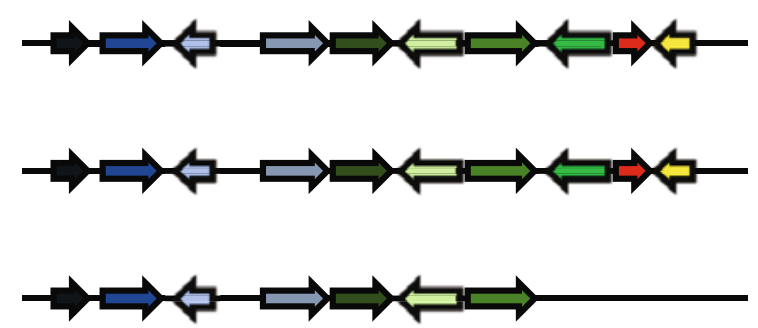

$\vdash$

$2.5 \mathrm{~kb}$
Fig. 2. Gene content and organization of the region containing the QTL marker associated with growth rate variation in Fusarium circinatum. Gene position and orientation are indicated with block arrows. Orange arrows illustrate genes only encoded in F. circinatum. Gene names are indicated below each species name. Similar colored genes illustrate shared collinearity and synteny. See Supplementary Table S4 for the predicted gene functions in $F$. circinatum. 
FCIRG_04559

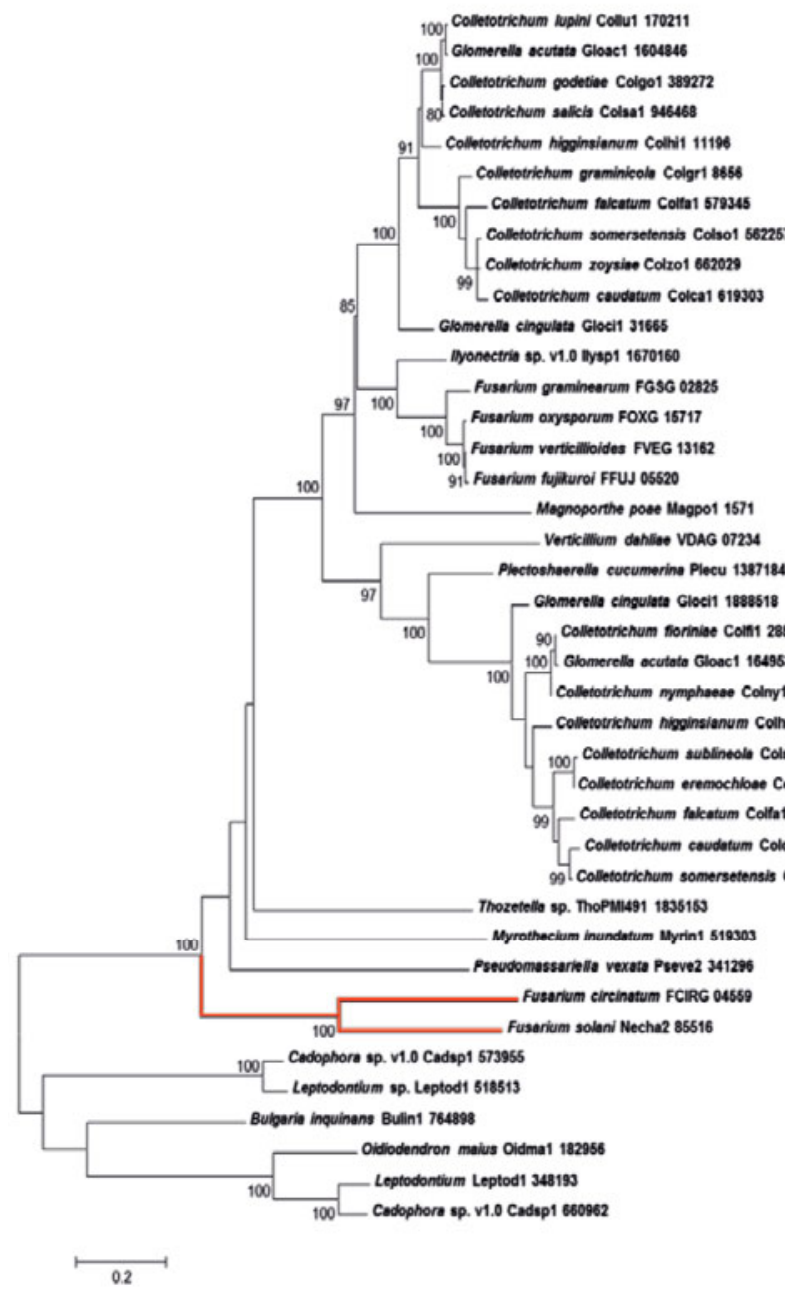

0.2

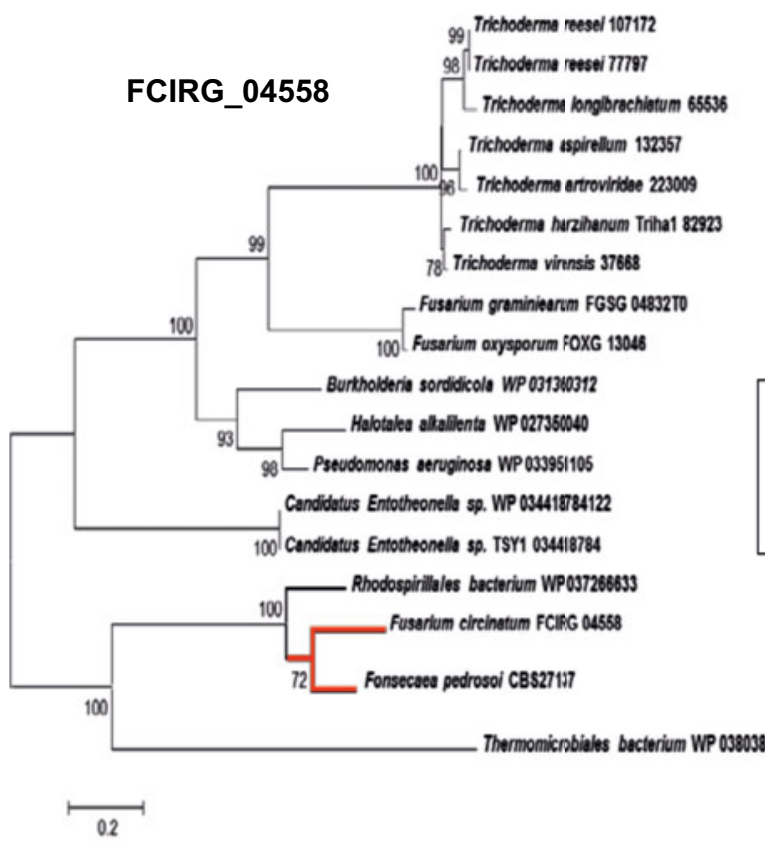

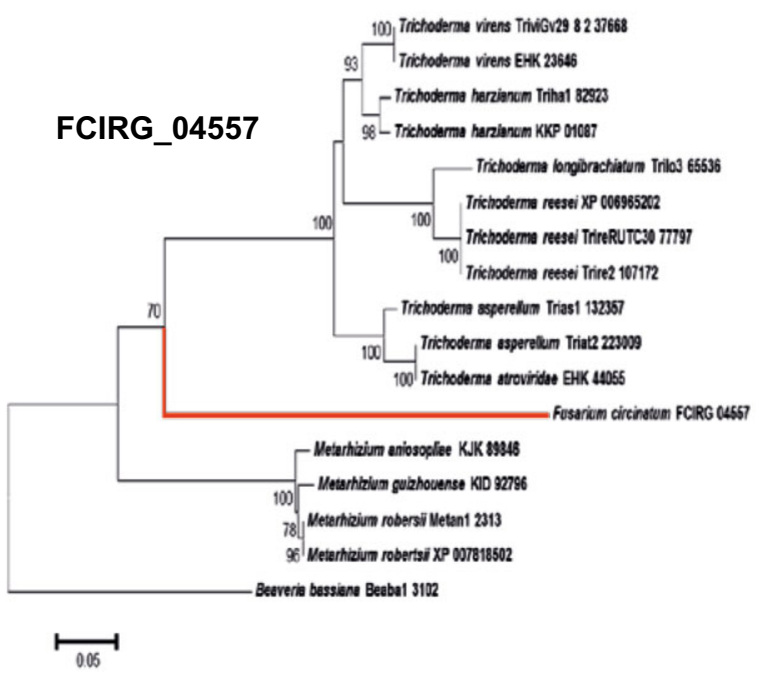

FCIRG_04555

B4, Trichoderma citrinovinidae Trici4 1200280 98 -Trichoderma longibrachiatum Trilo3 1392130 100 Trichoderme reesel Tires 110768 Trichoderma harzienum KK098440 Hypoxylon sp. CI4A 151722 Hypoxylon sp. HypEC38 1386672 100 Hypoxylon sp. HypCO275 1391925 Sporothrix brasillensis KIH 89839 100 Sporothrix schenckll ERS 97448 79 Sporothrix schenckii KJR 81948 M. inudatum Myrin1 512013

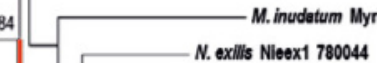

Sporothrix apiospermum KEZ 45657 Fusarium circinatum FCIRG 04555 Exophiala squamarina KEF 60516 Exophlala olligosperma KIW 37706 Bougainvillea spectabilis GAD 97227

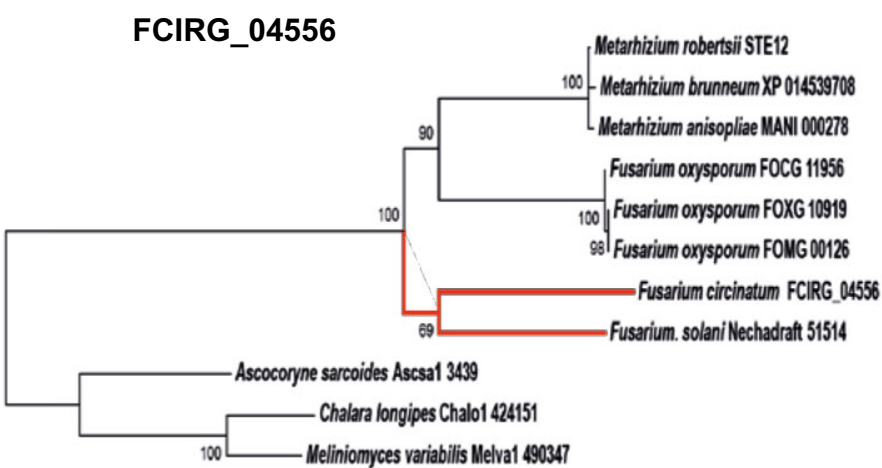

02

Fig. 3. Maximum likelihood trees constructed from the inferred Fusarium circinatum species-specific proteins FCIRG_04559, FCIRG_04558, FCIRG_04557, FCIRG_04556 and FCIRG_04555. Branches indicated in red show the position and closest relative or clade of $F$. circinatum in the five protein trees. Each alignment included only those protein sequences with $>40 \%$ amino acid similarity to that of the particular $F$. circinatum homologue. Bootstrap values $(>70 \%)$ are indicated at nodes, and the scale shows substitutions per site. 
and FCIRG_04557, these F. circinatum genes grouped with diverse non-Fusarium fungi. The results showed that FCIRG_04558 was nested within a bacterial clade. These results thus pointed towards HGT-based origins for the $F$. circinatum-specific 12000 bp region and its genes.

The non-vertical inheritance of the F. circinatum-specific region and its genes was also evident when we re-examined $\mathrm{G}+\mathrm{C}$ content. It was characterized by an average $\mathrm{G}+\mathrm{C}$ content of $51.2 \%$, which is significantly higher than the $47 \%$ in the rest of the FSP34 genome (Supplementary Table S16). A similar pattern was also observed for some of the individual genes (i.e., FCIRG_0556 and FCIRG_0559) (Supplementary Table S17-S18), but particularly pronounced in FCIRG_0558 (Supplementary Table S19-S20). This gene and its xenolog in F. pedrosoi (KIW 84299) had G+C contents exceeding 53 $\%$ (Supplementary Table S19), which supported the bacterial ancestry of this gene is dramatically different from the rest of their genomes.

\section{DISCUSSION}

The results of this study showed that the QTL-marker AT/AC $625 \mathrm{bh}$, which previously had been associated with growth rate (De Vos et al. 2011), is located on Chromosome 3 of $F$. circinatum. The genomic region underlying this marker is approximately $12000 \mathrm{bp}$ in size and is apparently unique to the species. It is absent from all of the examined genomes of other Fusarium species, including the closely related $F$. temperatum. It is, however, present in the genomes of all F. circinatum isolates we investigated, including the newly sequenced isolate $\mathrm{KS} 17$. The genomic regions directly adjacent to this unique region showed a high degree of synteny and collinearity across the FFSC and its sister taxa in the $F$. oxysporum species complex, but not in species more distantly related to the FFSC. This implies that the $F$. circinatum-specific gene region must have been introduced from elsewhere.

Detailed examination of the region up- and downstream of the F. circinatum-specific region suggested that it is located within Chromosome 3's subtelomere. This was evident from the high density of repeats and TEs that coincided with an AT-rich genomic environment. These genetic features are characteristic of distal subtelomeric regions (Flint et al. 1997, Cuomo et al. 2007, Wiemann et al. 2013, Chiara et al. 2015). In addition, the telomere-associated repeat motif, "TTAGGG", a known genetic feature of the distal parts of the telomeres (Garcia-Pedrajas \& Roncero 1996, Fulnečková et al. 2013), was prominent in this region. Similarly, synteny often also breaks down within subtelomeric regions, and these regions previously have been implicated in the development of species-specific adaptations and niche specification (Galagan et al. 2005, Moran et al. 2011, Zhao et al. 2014). Thus, the F. circinatum-specific 12000 bp located in a synteny break point is probably a consequence of the dynamic processes allowing genetic innovation in the telomeric regions of fungal chromosomes.

The genomic region in which the $F$. circinatum growth marker is located is predicted to be involved in producing proteins that have a diverse range of cellular, biological and metabolic functions. Previous studies on growth rate variation in $F$. circinatum and $F$. temperatum showed that $F$. circinatum grows significantly faster than $F$. temperatum at $25^{\circ} \mathrm{C}$ on solid media (De Vos et al. 2011). This QTL marker was also significantly correlated with growth rate variation amongst the $\mathrm{F} 1$ progeny of an interspecific cross between F. circinatum and F. temperatum (De Vos et al. 2011). In our study we showed that, comparable to the highlyvariable telomeric regions in F. fujikuroi isolates (Chiara et al. 2015), this genomic region is particularly enriched for genes involved in carbohydrate metabolism, metabolite transportation and transcriptional regulation. This adaption may have been brought about through the combination of enhanced substrate transport and carbon metabolism that is further supported by tight, species-specific transcriptional regulation (Proctor et al. 2009). Moreover, the clustering and possible co-regulation of these genes may assist this fungus to grow faster at higher temperatures (De Vos et al. 2011), in a species-specific manner.

Examination of the genetic makeup of the subtelomere of F. circinatum's Chromosome 3 allowed further insight regarding the evolution of such species-specific loci. Interspecific comparisons between homologous regions of $F$. circinatum and $F$. temperatum suggests that the differences in their TEs acquisition occurred in a species-specific manner. Transposable elements, specifically Retro- and DNA transposons, seemed to be confined to the supposed distal telomeric region of $F$. temperatum, whereas more TE integration in homologous $F$. circinatum regions continued into the adjacent telomere-proximal gene regions. Moreover, F. circinatum-specific TE acquisition also seemed to correlate with the location of the unique region. Previous studies established that $F$. circinatum and $F$. temperatum share a recent common ancestor (De Vos et al. 2014). Both the $F$. circinatum-specific TE acquisition and unique gene region were thus acquired after the divergence of these two species. It therefore stands to reason that the acquisition of the unique gene region probably involved a TE-mediated mechanism (see below). Future analysis of this region should seek to determine whether its acquisition coincided with (or potentially facilitated) the emergence of the pitch canker fungus as a separate species.

The introduction of a unique gene region into the $F$. circinatum genome may have been brought about by means of a number of possible mechanisms. It is generally thought that the repeat-rich nature of the distal and proximal telomeric regions of chromosomes frequently induce ectopic and nonhomologous recombination allowing for species-specific gene gains (Davière et al. 2001, Chow et al. 2012, Starnes et al. 2012). However, variable genomic regions may be more susceptible to TE invasion through non-homologous recombination. The more extensive, species-specific repeat sequences and TE acquisition within the telomeric-proximal region of $F$. circinatum may have facilitated such events allowing the species-specific gene gains within this region.

The findings of this study suggest that the genes encoded on the $F$. circinatum-specific region of Chromosome 3 did not result from internal duplications, but rather from HGT. These five genes have polyphyletic origins as they are derived from more than one independent evolutionary 
ancestor. Perhaps the most striking is gene FCIRG_04558 (encoding a class III aminotransferase) that share a recent common ancestor with bacteria. In fact, our data suggest that only two independent introductions of a FCIRG_04558 homolog have so far occurred in fungi (i.e., into unrelated lineages represented by $F$. pedrosoi [Eurotiomycetes] and F. circinatum [Sordariomycetes]). Also, the speciesspecific genes showed marked differences in $\mathrm{G}+\mathrm{C}$ content compared to that of the surrounding gene regions, the rest of Chromosome 3, and the remainder of the F. circinatum genome. Interestingly, the lack of introns in the F. pedrosoi gene, together with the higher $\mathrm{G}+\mathrm{C}$ content, would also fit the scenario of bacterial ancestry implied by the phylogeny. These findings are thus in line with the view that similarities in nucleotide composition of xenologs reflect features of both donor and recipient genomes involved in HGT (Lawrence \& Ochman 1998).

This study has provided new insights into the origin and evolution of genes encoded within a locus implicated in growth rate regulation of the pitch canker fungus $F$. circinatum (De Vos et al. 2011). A main hypothesis emerging from our work is that the dynamic evolutionary processes associated with subtelomeric regions likely facilitated the emergence of the $F$. circinatum-specific sequence, which in turn enabled differentiation and adaptation of the fungus in a speciesspecific manner. Details regarding the precise evolutionary mechanisms involved in the origin of this F. circinatumspecific locus might become apparent when the genomes of Fusarium species with more recent common ancestry to that of $F$. circinatum are investigated. Additionally, establishing the functional relevance of each of the species-specific proteins identified in this study will be the focus of future studies.

\section{ACKNOWLEDGMENTS}

This work is based on research funded and supported in part by the South African National Department of Science and Technology (DST), National Research Foundation (NRF) and Technology and Human Resources of Industry Programme (THRIP), as well as the University of Pretoria and the Tree Protection Cooperative Programme (TPCP). The Grant holders acknowledge that opinions, findings and conclusions or recommendations expressed in any publication generated by NRF supported research are that of the author(s) and that the NRF accepts no liability whatsoever in this regard.

\section{REFERENCES}

Abascal F, Zardoya R, Posada D (2005) ProtTest: selection of best-fit models of protein evolution. Bioinformatics 21: 2104-2105.

Altschul SF, Madden TL, Schäffer AA, Zhang JJ, Zhang Z, et al. (1997) Gapped BLAST and PSI-BLAST: a new generation of protein database search programs. Nucleic Acids Research 25: 3389-3402.

Alves FL, Ribeiro MA, Hahn RC, DeMelo M,Teixeira M, De Camargo ZP, et al. (2014) Transposable elements and two other molecular markers as typing tools for the genus Paracoccidioides. Medical Mycology: myu074.
Behnsen J, Hartmann A, Schmaler J, Gehrke A, Brakhage AA, et al. (2008) The opportunistic human pathogenic fungus Aspergillus fumigatus evades the host complement system. Infection and Immunity 76: 820-827.

Benson G (1999) Tandem repeats finder: a program to analyze DNA sequences. Nucleic Acids Research 27: 573-580.

Blin K, Medema MH, Kazempour D, Fischbach MA, Breitling R, et al. (2013) antiSMASH 2.0-a versatile platform for genome mining of secondary metabolite producers. Nucleic Acids Research: gkt449.

Boetzer M, Pirovano W (2012) Towards almost closed genomes with GapFiller. Genome Biology 13: R56.

Braun EL, Halpern AL, Nelson MA, Natvig DO (2000) Large-scale comparison of fungal sequence information: mechanisms of innovation in Neurospora crassa and gene loss in Saccharomyces cerevisiae. Genome Research 10: 416-430.

Brown JR, Doolittle WF (1999) Gene descent, duplication, and horizontal transfer in the evolution of glutamyl-and glutaminyltRNA synthetases. Journal of Molecular Evolution 49: 485-495.

Chiara M, Fanelli F, Mulè G, Logrieco AF, Pesole G, et al. (2015) Genome sequencing of multiple isolates highlights subtelomeric genomic diversity within Fusarium fujikuroi. Genome Biology and Evolution 7: 3062-3069.

Chow EW, Morrow CA, Djordjevic JT, Wood IA, Fraser JA (2012) Microevolution of Cryptococcus neoformans driven by massive tandem gene amplification. Molecular Biology and Evolution 29: 1987-2000.

Chuma I, Isobe C, Hotta Y, Ibaragi K, Futamata N, et al. (2011) Multiple translocation of the AVR-Pita effector gene among chromosomes of the rice blast fungus Magnaporthe oryzae and related species. PLoS Pathogens 7: e1002147.

Coleman JJ, Rounsley SD, Rodriguez-Carres M, Kuo A, Wasmann CC, et al. (2009) The genome of Nectria haematococca: contribution of supernumerary chromosomes to gene expansion. PLoS Genetics 5: e1000618.

Crisp A, Boschetti C, Perry M, Tunnacliffe A, Micklem G (2015) Expression of multiple horizontally acquired genes is a hallmark of both vertebrate and invertebrate genomes. Genome Biology 16: 50 .

Cuomo CA, Güldener U, Xu JR, Trail F, Turgeon BG, et al. (2007) The Fusarium graminearum genome reveals a link between localized polymorphism and pathogen specialization. Science 317: 1400-1402.

Daboussi MJ, Capy P (2003) Transposable elements in filamentous fungi. Annual Reviews in Microbiology 57: 275-299.

Davière, JM, Langin T, Daboussi MJ (2001) Potential role of transposable elements in the rapid reorganization of the Fusarium oxysporum genome. Fungal Genetics and Biology 34: 177-192.

De Vos, L, Myburg AA, Wingfield MJ, Desjardins AE, Gordon TR, et al. (2007) Complete genetic linkage maps from an interspecific cross between Fusarium circinatum and Fusarium subglutinans. Fungal Genetics and Biology 44: 701-714.

De Vos L, Van der Nest MA, Van der Merwe NA, Myburg AA, Wingfield MJ, et al. (2011) Genetic analysis of growth, morphology and pathogenicity in the $\mathrm{F} 1$ progeny of an interspecific cross between Fusarium circinatum and Fusarium subglutinans. Fungal Biology 115: 902-908

De Vos, L, Steenkamp ET, Martin SH, Santana, QC, Fourie G, et al. (2014) Genome-wide macrosynteny among Fusarium species in 
the Gibberella fujikuroi complex revealed by amplified fragment length polymorphisms. PloS One 9: e114682.

Desjardins A, Plattner R, Gordon TR (2000) Gibberella fujikuroi mating population $A$ and Fusarium subglutinans from teosinte species and maize from Mexico and Central America. Mycological Research 104: 865-72.

Fedorova ND, Khaldi N, Joardar VS, Maiti R, Amedeo P, et al. (2008) Genomic islands in the pathogenic filamentous fungus Aspergillus fumigatus. PLoS Genetics 4: e1000046.

Flint J, Thomas K, Micklemz G, Raynhaml H, Clarkl K, et al. (1997) The relationship between chromosome structure and function at a human telomeric region. Nature Genetics 15: 252-257.

Fulnečková, J, Ševčíková T, Fajkus J, Lukešová A, Lukeš M, et al. (2013) A broad phylogenetic survey unveils the diversity and evolution of telomeres in eukaryotes. Genome Biology and Evolution 5: 468-483.

Gac M, Giraud T (2008) Existence of a pattern of reproductive character displacement in Homobasidiomycota but not in Ascomycota. Journal of Evolutionary Biology 21: 761-772.

Galagan JE, Calvo SE, Cuomo C, Ma L-J, Wortman JR, et al. (2005) Sequencing of Aspergillus nidulans and comparative analysis with $A$. fumigatus and A. oryzae. Nature 438: 1105-1115.

Garcia-Pedrajas MD, Roncero MIG (1996) A homologous and self-replicating system for efficient transformation of Fusarium oxysporum. Current Genetics 29: 191-198.

Gardiner DM, Kazan K, Manners JM (2013) Cross-kingdom gene transfer facilitates the evolution of virulence in fungal pathogens. Plant Science 210: 151-158.

Geiser DM, Aoki T, Bacon CW, Baker SE, Bhattacharyya MK, et al. (2013) One fungus, one name: defining the genus Fusarium in a scientifically robust way that preserves longstanding use. Phytopathology 103: 400-408.

Glenn AE, Davis CB, Gao M, Gold SE, Mitchell TR, et al. (2016) Two horizontally transferred xenobiotic resistance gene clusters associated with detoxification of benzoxazolinones by Fusarium species. PloS One 11: e0147486.

Goodwin SB, M'barek SB, Dhillon,B Wittenberg AH, Crane CF, et al. (2011) Finished genome of the fungal wheat pathogen Mycosphaerella graminicola reveals dispensome structure, chromosome plasticity, and stealth pathogenesis. PLoS Genetics 7: e1002070.

Grigoriev IV, Nikitin R, Haridas S, Kuo A, Ohm R, et al. (2013) MycoCosm portal: gearing up for 1000 fungal genomes. Nucleic Acids Research 42: D699-04.

Hall TA (1999) BioEdit: a user-friendly biological sequence alignment editor and analysis program for Windows 95/98/NT. Nucleic Acids Symposium Series 41: 95-98

Hansen FT, Gardiner DM, Lysøe E, Fuertes PR, Tudzynski B, et al. (2015) An update to polyketide synthase and non-ribosomal synthetase genes and nomenclature in Fusarium. Fungal Genetics and Biology 75: 20-29.

Hepting GH, Roth ER (1946) Pitch canker, a new disease of some southern pines. Journal of Forestry 44: 742-744.

Herron DA, Wingfield MJ, Wingfield BD, Rodas C, Marincowitz S, et al. (2015) Novel taxa in the Fusarium fujikuroi species complex from Pinus spp. Studies in Mycology 80: 131-150.

Hoff KJ, Stanke M (2013) WebAUGUSTUS - a web service for training AUGUSTUS and predicting genes in eukaryotes. Nucleic Acids Research 41: W123-128.

Jaramillo VD, Sukno SA, Thon MR (2015) Identification of horizontally transferred genes in the genus Colletotrichum reveals a steady tempo of bacterial to fungal gene transfer. BMC Genomics 16: 2.

Kohany O, Gentles AJ, Hankus L, Jurka J (2006) Annotation, submission and screening of repetitive elements in Repbase: RepbaseSubmitter and Censor. BMC Bioinformatics 7: 474.

Lawrence JG, Ochman H (1998) Molecular archaeology of the Escherichia coli genome. Proceedings of the National Academy of Sciences, USA 95: 9413-9417.

Leslie JF, Summerell BA (2006) The Fusarium Laboratory Manual. Ames, lowa: Wiley Online Library.

Li W, Cowley A, Uludag M, Gur T, McWilliam H, et al. (2015) The EMBL-EBI bioinformatics web and programmatic tools framework. Nucleic Acids Research: gkv279.

Lysøe E, Harris LJ, Walkowiak S, Subramaniam R, Divon HH, et al. (2014) The genome of the generalist plant pathogen Fusarium avenaceum is enriched with genes involved in redox, signaling and secondary metabolism. PloS One 9: e112703.

Ma L-J, Van der Does HC, Borkovich KA, Coleman JJ, Daboussi M.-J, et al. (2010) Comparative genomics reveals mobile pathogenicity chromosomes in Fusarium. Nature 464: 367-373.

Möller E, Bahnweg G, Sandermann H, Geiger H (1992) A simple and efficient protocol for isolation of high molecular weight DNA from filamentous fungi, fruit bodies, and infected plant tissues. Nucleic Acids Research 20: 6115-6116.

Moran GP, Coleman DC, Sullivan DJ (2011) Comparative genomics and the evolution of pathogenicity in human pathogenic fungi. Eukaryotic Cell 10: 34-42.

Nelson KE, Clayton RA, Gill SR, Gwinn ML, Dodson RJ, et al. (1999) Evidence for lateral gene transfer between Archaea and bacteria from genome sequence of Thermotoga maritima. Nature 399: 323-329.

Niehaus EM, Münsterkötter M, Proctor RH, Brown DW, Sharon A, et al. (2016) Comparative "omics" of the Fusarium fujikuroi species complex highlights differences in genetic potential and metabolite synthesis. Genome Biology and Evolution 8: 3574-3599.

O'Donnell K, Rooney AP, Proctor RH, Brown DW, McCormick SP, et al. (2013) Phylogenetic analyses of RPB1 and RPB2 support a middle Cretaceous origin for a clade comprising all agriculturally and medically important fusaria. Fungal Genetics and Biology 52: 20-31.

Papadopoulos JS, Agarwala R (2007) COBALT: constraint-based alignment tool for multiple protein sequences. Bioinformatics 23: 1073-1079.

Proctor RH, McCormick SP, Alexander NJ, Desjardins AE (2009) Evidence that a secondary metabolic biosynthetic gene cluster has grown by gene relocation during evolution of the filamentous fungus Fusarium. Molecular Microbiology 74: 1128-1142.

Raffaele S, Kamoun S (2012) Genome evolution in filamentous plant pathogens: why bigger can be better. Nature Reviews Microbiology 10: 417-430.

Rombauts S, Van de Peer Y, Rouzé P (2003) AFLPinSilico, simulating AFLP fingerprints. Bioinformatics 19: 776-777.

Scauflaire J, Gourgue M, Munaut F (2011) Fusarium temperatum sp. nov. from maize, an emergent species closely related to Fusarium subglutinans. Mycologia 103: 586-597.

Sieber CM, Lee W, Wong P, Münsterkötter M, Mewes HW, et al. (2014) The Fusarium graminearum genome reveals more secondary metabolite gene clusters and hints of horizontal gene transfer. PloS One 9: e110311. 
Simão FA, Waterhouse RM, loannidis P, Kriventseva EV, Zdobnov EM (2015) BUSCO: assessing genome assembly and annotation completeness with single-copy orthologs. Bioinformatics 31 : 3210-3212.

Simpson JT, Wong K, Jackman SD, Schein JE, Jones SJM, et al. (2009) ABySS: A parallel assembler for short read sequence data. Genome Research 19: 1117-1123.

Skamnioti P, Furlong RF, Gurr SJ (2008) Evolutionary history of the ancient cutinase family in five filamentous ascomycetes reveals differential gene duplications and losses and in Magnaporthe grisea shows evidence of sub-and neo-functionalization. New Phytologist 180: 711-721.

Spanu PD, Abbott JC, Amselem J, Burgis TA, Soanes DM, et al. (2010) Genome expansion and gene loss in powdery mildew fungi reveal tradeoffs in extreme parasitism. Science 330: 15431546.

Sperschneider J, Gardiner DM, Thatcher LF, Lyons R, Singh KB, et al. (2015) Genome-wide analysis in three Fusarium pathogens identifies rapidly evolving chromosomes and genes associated with pathogenicity. Genome Biology and Evolution: evv092.

Starnes JH, Thornbury DW, Novikova OS, Rehmeyer CJ, Farman ML (2012) Telomere-targeted retrotransposons in the rice blast fungus Magnaporthe oryzae: agents of telomere instability. Genetics 191: 389-406.

Steenkamp ET, Makhari OM, Coutinho TA, Wingfield BD, Wingfield MJ (2014) Evidence for a new introduction of the pitch canker fungus Fusarium circinatum in South Africa. Plant Pathology 63: 530-538.

Steenkamp ET, Wingfield BD, Coutinho TA, Wingfield MJ, Marasas WFO (1999) Differentiation of Fusarium subglutinans f. sp. pini by histone gene sequence data. Applied and Environmental Microbiology 65: 3401-3406.

Stewart JE, Abdo Z, Glenn AE (2014) Gene clusters FDB1 and FDB2 in Fusarium verticillioides were acquired through multiple horizontal gene transfer events. Phythopathology 104: 114
Tamura K, Stecher G, Peterson D, Filipski A, Kumar S (2013) MEGA6: molecular evolutionary genetics analysis version 6.0. Molecular Biology and Evolution 30: 2725-2729.

Tarailo-Graovac M, Chen N (2009) Using RepeatMasker to identify repetitive elements in genomic sequences. Current Protocols in Bioinformatics: 4.10: 11-14.

Untergasser A, Cutcutache I, Koressaar T, Ye J, Faircloth BC, et al. (2012) Primer3 - new capabilities and interfaces. Nucleic Acids Research 40: e115.

Van der Nest MA, Beirn LA, Crouch JA, Demers JE, De Beer, ZW, et al. (2014) IMA Genome-F 3: Draft genomes of Amanita jacksonii, Ceratocystis albifundus, Fusarium circinatum, Huntiella omanensis, Leptographium procerum, Rutstroemia sydowiana, and Sclerotinia echinophila. IMA Fungus 5: 473-486.

Waalwijk C, Van der Lee T, De Vries, I, Hesselink T, Arts J, et al. (2004) Synteny in toxigenic Fusarium species: the fumonisin gene cluster and the mating type region as examples. European Journal of Plant Pathology 110: 533-544.

Wiemann P, Sieber CM, Von Bargen KW, Studt L, Niehaus EM, et al. (2013) Deciphering the cryptic genome: genome-wide analyses of the rice pathogen Fusarium fujikuroi reveal complex regulation of secondary metabolism and novel metabolites. PLoS Pathogens 9: e1003475.

Wingfield BD, Steenkamp ET, Santana QC, Coetzee MPA, Bam $S$, et al. (2012) First fungal genome sequence from Africa: a preliminary analysis. South African Journal of Science 108: 1-2.

Wisecaver JH, Slot JC, Rokas A (2014) The evolution of fungal metabolic pathways. PLoS Genetics 10: e1004816.

Zdobnov EM, Apweiler R (2001) InterProScan-an integration platform for the signature-recognition methods in InterPro. Bioinformatics 17: 847-848.

Zhao C, Waalwijk C, De Wit PJ, Tang D, Van der Lee T (2014) Relocation of genes generates non-conserved chromosomal segments in Fusarium graminearum that show distinct and coregulated gene expression patterns. BMC Genomics 15: 191. 